\title{
Two-photon parametric pumping versus two-photon absorption: A quantum jump approach
}

\author{
E. S. Guerra, ${ }^{*}$ B. M. Garraway, and P. L. Knight \\ Optics Section, The Blackett Laboratory, Imperial College, Prince Consort Road, London SW7 2BZ, United Kingdom
}

(Received 7 November 1996)

\begin{abstract}
We show that it is possible to produce superpositions of distinct coherent states (even or odd coherent states) in a cavity where the field is pumped by two-photon parametric amplification and simultaneously undergoes two-photon absorption by a beam of three-level atoms that travel through the cavity interacting with the cavity field. Previous studies modeled the absorber with an effective Hamiltonian without involving real atomic excitation or entanglement, a procedure justified only for weak coupling of the two-photon absorbing atoms. We examine the validity of this assumption by modeling the atomic absorber dynamics from the onset. In order to study the system numerically we make use of a Monte Carlo wave-function method in which the two-photon absorbing atoms can interact with the cavity and evolve with large Rabi angles. [S1050-2947(97)01405-4]
\end{abstract}

PACS number(s): 42.50.Dv, 42.50.Lc

\section{INTRODUCTION}

The interaction of matter and radiation can change the statistical properties of the electromagnetic field and generate fields with special quantum features. Nonlinear processes involving two-photon transitions play an important role in the production of nonclassical light and have long been utilized as a way of producing squeezed light [1]. In particular twophoton parametric pumping has been shown to be an important source of squeezed light [2]. It has also been shown that interactions involving two-photon transitions can generate or preserve quantum features. For example, the one-photon absorption process (a linear interaction) destroys quantum features of the field rapidly, whereas two-photon absorption processes (a nonlinear interaction) can build up quantum features [3].

The two-photon absorption process that a field undergoes when traveling through a two-photon absorbing medium has been studied in Refs. [3-6]. The time evolution of the field reduced density matrix for such a process is described by a master equation where the change in the field reduced density matrix is obtained by applying a Liouvillian superoperator to the initial reduced density matrix of the field [4]. The analytical solution of this master equation has been obtained [3] and the dynamic behavior as well as the steady state of the system has been studied. If a field is pumped by a twophoton parametric process and the losses are due only to two-photon absorption then, depending on the initial state, the field can evolve into Schrödinger cat states, specifically the even coherent state and the odd coherent state $[5,6]$. The dynamics of these systems was studied in detail in Refs. $[5,6]$.

Recent technological achievements have made possible the construction of electromagnetic cavities with very high quality factors $(Q)$ [7]. This has led to the development of cavity QED, and many interesting problems that in the past were mere theoretical idealizations now are perfectly pos-

\footnotetext{
*Present address: Instituto de Fisica, Universidade Federal do Rio de Janeiro, Caixa Postal 68528, Rio de Janeiro BR-21945-970, Rio de Janeiro, Brazil.
}

sible to realize experimentally. An example is the possibility of manipulating intracavity fields and of producing fields with special quantum features. The interaction of a single atom with a single quantized cavity field mode has been much studied [8] and is one particular way of building up such fields. Micromasers [9] and microlasers [10] are experimental realizations of such systems. In those systems the high cavity quality factor $(Q)$ permits the production of fields with very peculiar, or quantum, features and with only one atom interacting with the cavity field at a time. This is quite contrary to a typical laser or a system in which the field propagates through two-photon absorbing media, where we have a large number of atoms interacting with the field. A further example of a situation in which "two-photon" (actually "two-phonon") processes can dominate is that of a laser-cooled, trapped ion with vibrational states that can be manipulated using sequences of $\pi$ pulses driving vibrational sidebands. For such ionic motions dissipation is very weak, and in this way cavity QED can be realized without a cavity [11]. Indeed it is straightforward to translate cavity QED results (such as ours) into a direct ion trap equivalent, which may well be easier to realize $[12,13]$.

Our aim in this paper is to study the field produced inside a cavity pumped by a two-photon parametric process and subjected to two-photon absorption. The absorption results in the relaxation of the cavity field and is caused by a stream of three-level atoms that enter the cavity in the lower state. Figure 1 shows a sketch of the kind of system we will study. Previous studies modeled the absorber with an effective Hamiltonian without involving real atomic excitation or entanglement, a procedure justified only for weak coupling. We will examine the validity of this assumption by modeling the detail of the atomic absorber dynamics from the outset. We assume that the cavity has an extremely high $Q$ factor and we neglect one-photon losses, although such losses can easily be included. Generally, one-photon losses destroy the quantum features that we seek to demonstrate here. For the case of weak coupling to the three-level atoms, we have already studied these detrimental effects in Ref. [6]. We will not pursue the (realistic) inclusion of these processes here in order to focus on the principal results from our model. Thus we assume that the atoms do not decay during their passage 

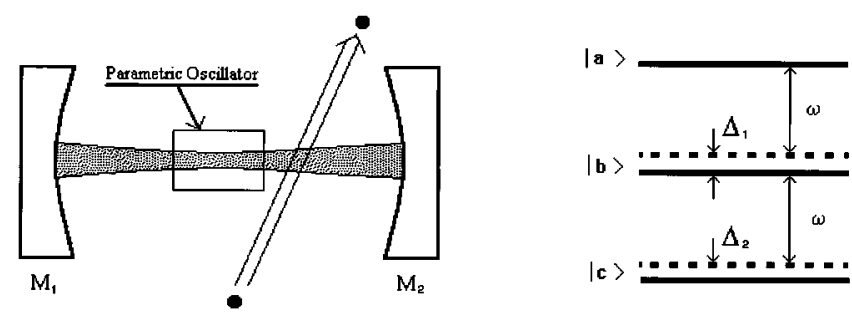

FIG. 1. The system considered in this paper consists of a parametric oscillator driving a single-mode cavity field that is additionally pumped by a beam of three-level atoms that crosses the cavity as shown on the left-hand side of the figure. The parametric driving will try to create a squeezed state in the cavity, while the beam of atoms provides a dissipative mechanism of an unusual kind. On the right-hand side we show the energy-level scheme with atomic levels $|a\rangle,|b\rangle$, and $|c\rangle$ and detunings $\Delta_{1}$ and $\Delta_{2}$.

through the cavity. Therefore, the mean lifetime of the atoms must be much larger than the interaction time. As we will see it is possible to generate Schrödinger cat states in such systems.

The outline of this paper is as follows. In Sec. II we obtain the master equation for the two-photon absorption process. In Sec. III we take into account two-photon parametric pumping and show that the competition between the parametric pumping and the two-photon absorption generates a steady-state field that is an odd or an even coherent state if the coupling constant of the two-photon absorbing atoms are small. In Sec. IV we relax the assumption of small Rabi angles for the two-photon absorbing atoms and derive a more general master equation, which becomes the focus for the rest of the paper. In Sec. V we show how the master equation obtained in Sec. IV can be studied using a Monte Carlo quantum jump approach $[14,15]$. In Sec. VI we present and interpret our numerical results. Finally, in Sec. VII we conclude and summarize our results.

\section{TWO-PHOTON ABSORPTION MASTER EQUATION}

The two-photon absorption master equation for light traveling through a two-photon absorbing medium has been derived in Ref. [4]. Here we review the derivation of this master equation starting from a simple two-photon Hamiltonian for an absorption process by a stream of atoms that pass through a cavity inside which we have a single dominant mode. The states $|a\rangle$ and $|c\rangle$ will stand for the upper and lower atomic levels, respectively. The Hamiltonian for the system may be written as

$$
\begin{aligned}
H= & \hbar \omega A^{\dagger} A+\frac{\hbar}{2} \omega_{a c}(|a\rangle\langle a|-| c\rangle\langle c|) \\
& +\hbar \lambda\left(A^{2}|a\rangle\left\langle c\left|+A^{\dagger 2}\right| c\right\rangle\langle a|\right) \equiv H_{0}+H_{I},
\end{aligned}
$$

where $A$ and $A^{\dagger}$ are the field annihilation and creation operators. We note the absence of a Stark shift in this phenomenological Hamiltonian. If we write

$$
V_{I}=e^{i H_{0} t / \hbar} H_{I} e^{-i H_{0} t / \hbar}
$$

we have

$$
V_{I}=\hbar \lambda\left(A^{2}|a\rangle\left\langle c\left|+A^{\dagger 2}\right| c\right\rangle\langle a|\right),
$$

where we have assumed $\omega_{a c}=2 \omega$. We expand the timedependent state vector in the superposition

$\left|\psi\left(t_{i}+t_{\text {int }}\right)\right\rangle=\sum_{N}\left[C_{a, N}\left(t_{i}+t_{\text {int }}\right)|a\rangle+C_{c, N}\left(t_{i}+t_{\text {int }}\right)|c\rangle\right]|N\rangle$,

where $t_{i}$ is the time at which the $i$ th atom enters the cavity and $t_{\text {int }}$ is the time the atom has spent in the cavity.

The derivation of the master equation (5) below can be made by following the method used to obtain the one-photon loss process master equation presented in Ref. [16]. By solving the Schrödinger equation and making the assumption of small Rabi angles, in order to expand the amplitude coefficients in the vector state of the atom-field system, we obtain

$$
\begin{aligned}
\dot{\rho}(t) & =r L_{c} \rho(t) \\
& =K_{L}\left\{2 A^{2} \rho(t) A^{\dagger 2}-A^{\dagger 2} A^{2} \rho(t)-\rho(t) A^{\dagger 2} A^{2}\right\},
\end{aligned}
$$

where $r$ is the average rate of atomic injection and we have defined the constant

$$
K_{L}=\frac{r \lambda^{2} t_{\mathrm{int}}^{2}}{2}
$$

where the constant $K_{L}$ is related to the two-photon absorption coefficient $[5,6]$.

The two-photon absorption master equation (5) above was obtained from a simple model in which we have assumed the field in the cavity interacts with a beam of two-level atoms that undergo small changes in the Rabi angle. Now we consider the more realistic situation in which the atomic beam source of absorption is properly modeled by three-level atoms (such that two-photon interactions are dominant) and where we do not assume small Rabi angles. We will still assume that the density of the atomic beam is sufficiently low in order that only one atom interacts with the field at a time.

In this more detailed model, a flux of three-level atoms, with states $|a\rangle,|b\rangle$, and $|c\rangle$, is injected into a cavity tuned to the frequency $\omega$. The atomic states $|a\rangle$ and $|c\rangle$ are the upper and lower states as above and $|b\rangle$ is an intermediate state with energy $\hbar \omega_{b}$ nearly halfway between the energies $\hbar \omega_{a}$ and $\hbar \omega_{c}$. We again assume that the atoms are injected into the cavity with an average rate $r$ and that the atomic beam is monoenergetic, with the atoms spending a time $t_{\text {int }}$ inside the cavity (see Fig. 1). The detunings $\Delta_{1}=\omega_{a b}-\omega=\omega_{a}-\omega_{b}$ $-\omega$ and $\Delta_{2}=\omega_{b c}-\omega=\omega_{b}-\omega_{c}-\omega$ are chosen in such way as to enhance the two-photon transition probability while at the same time keeping negligible the resonant one-photon cascade $a \rightarrow b \rightarrow c$.

The Hamiltonian that describes the dynamics of this three-level system in the rotating-wave approximation is given by 


$$
\begin{aligned}
H= & \hbar \omega A^{\dagger} A+\hbar \omega_{a}|a\rangle\left\langle a\left|+\hbar \omega_{b}\right| b\right\rangle\langle b| \\
& +\hbar \omega_{c}|c\rangle\langle c|+\hbar g_{a b}\left(A|a\rangle\left\langle b\left|+A^{\dagger}\right| b\right\rangle\langle a|\right) \\
& +\hbar g_{b c}\left(A|b\rangle\left\langle c\left|+A^{\dagger}\right| c\right\rangle\langle b|\right),
\end{aligned}
$$

where $g_{a b}\left(g_{b c}\right)$ are the one-photon Rabi frequencies related to the interactions between the levels $a$ and $b$ ( $b$ and $c$ ) and the operator $A\left(A^{\dagger}\right)$ is the annihilation (creation) operator for the electromagnetic field mode of frequency $\omega$. In the interaction picture the Hamiltonian becomes

$$
V_{I}=\hbar g_{a b} e^{i \Delta_{1} \tau} A|a\rangle\left\langle b\left|+\hbar g_{b c} e^{i \Delta_{2} \tau} A\right| b\right\rangle\langle c|+\text { H.c. }
$$

Then the state of the system at an instant $t_{i}+\tau$ can be written in the form

$$
\begin{aligned}
\left|\Psi\left(t_{i}+\tau\right)\right\rangle= & \sum_{N}\left[C_{a, N}\left(t_{i}+\tau\right)|a\rangle+C_{b, N}\left(t_{i}+\tau\right)|b\rangle\right. \\
& \left.+C_{c, N}\left(t_{i}+\tau\right)|c\rangle\right]|N\rangle .
\end{aligned}
$$

Substituting this state in the Schrödinger equation, we obtain

$$
\begin{array}{r}
\dot{C}_{a, N}\left(t_{i}+\tau\right)=-i g_{a b} e^{i \Delta_{1} \tau} \sqrt{N+1} C_{b, N+1}\left(t_{i}+\tau\right), \\
\dot{C}_{b, N+1}\left(t_{i}+\tau\right)=-i g_{a b} e^{-i \Delta_{1} \tau} \sqrt{N+1} C_{a, N}\left(t_{i}+\tau\right) \\
-i g_{b c} e^{i \Delta_{2} \tau} \sqrt{N+2} C_{c, N+2}\left(t_{i}+\tau\right), \\
\dot{C}_{c, N+2}\left(t_{i}+\tau\right)=-i g_{b c} e^{-i \Delta_{2} \tau} \sqrt{N+2} C_{b, N+1}\left(t_{i}+\tau\right),
\end{array}
$$

where the overdot represents the derivative $d / d \tau$ and $t_{i}$ is the time at which the $i$ th atom enters the cavity.

It is possible to solve the system of differential equations (10) approximately and to obtain the field reduced density matrix in the number representation after the passage of a single atom through the cavity. In Appendix A we show that we can do this if we make the assumption

$$
\begin{aligned}
& \frac{g_{a b}}{g_{b c}}=\frac{g_{a b}}{g}=1+\epsilon^{2}, \\
& \frac{\Delta_{1}}{\Delta_{2}}=\frac{\Delta}{\Delta_{2}}=-1+\epsilon^{2},
\end{aligned}
$$

where $\epsilon=g / \Delta$ and

$$
\frac{g^{2}}{\Delta} \ll \Delta .
$$

If we make use of the approximate solution of Eq. (10) obtained in Appendix A [see Eqs. (A33)], the field reduced density matrix after the passage of one atom through the cavity is given by

$$
\begin{aligned}
\rho_{N, M}\left(t_{i}+t_{\text {int }}\right)= & e^{-i(N-M)\left(g^{2} / \Delta\right) t_{\text {int }}\left[\sin \left(\Theta_{N} \frac{t_{\text {int }}}{2}\right)\right.} \\
& \times \sin \left(\Theta_{M} \frac{t_{\text {int }}}{2}\right) \rho_{N+2, M+2}\left(t_{i}\right) \\
& \left.+\cos \left(\Theta_{N-2} \frac{t_{\text {int }}}{2}\right) \cos \left(\Theta_{M-2} \frac{t_{\text {int }}}{2}\right) \rho_{N, M}\left(t_{i}\right)\right],
\end{aligned}
$$

where the generalized Rabi frequency $\Theta_{N}$ is given by

$$
\Theta_{N}=2 \sqrt{(N+1)(N+2)} \frac{g^{2}}{\Delta} .
$$

For the populations we have

$$
\rho_{N, N}\left(t_{i}+t_{\text {int }}\right)=\alpha_{N} \rho_{N+2, N+2}\left(t_{i}\right)+\left(1-\alpha_{N-2}\right) \rho_{N, N}\left(t_{i}\right),
$$

where

$$
\alpha_{N}=\sin ^{2}\left(\Theta_{N} \frac{t_{\text {int }}}{2}\right) .
$$

In the remainder of this section we focus on the weakcoupling limit where we can establish the connection with the master equation (5). We write $g^{2} / \Delta=\lambda$ and expand $\sin \left(\Theta_{N} t_{\text {int }} / 2\right)$ up to first order and $\cos \left(\Theta_{N} t_{\text {int }} / 2\right)$ up to second order to obtain

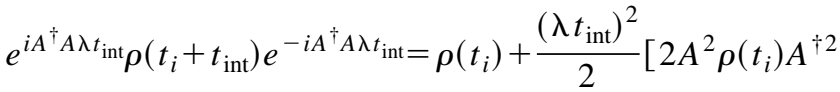

$$
\begin{aligned}
& \left.-A^{\dagger 2} A^{2} \rho\left(t_{i}\right)-\rho\left(t_{i}\right) A^{\dagger 2} A^{2}\right]
\end{aligned}
$$

Now, if we multiply both sides of Eq. (17) by $e^{i A^{\dagger} A \lambda t_{i}}$ on the left and $e^{-i A^{\dagger} A \lambda t_{i}}$ on the right and define

$$
e^{i A^{\dagger} A \lambda t} \rho(t) e^{-i A^{\dagger} A \lambda t}=\widetilde{\rho}(t),
$$

we can follow the same procedure as in Ref. [16] and set $t=t_{i}$ so that

$$
\bar{\Delta} \widetilde{\rho}(t)=\bar{\rho}(t+\Delta t)-\bar{\rho}(t)=r \Delta t L_{c} \overline{\widetilde{\rho}(t)} .
$$

Then, by dropping the overbar so as to simplify notation, we obtain

$$
\frac{d \widetilde{\rho}(t)}{d t}=K_{L}\left[2 A^{2} \widetilde{\rho}(t) A^{\dagger 2}-A^{\dagger 2} A^{2} \widetilde{\rho}(t)-\widetilde{\rho}(t) A^{\dagger 2} A^{2}\right],
$$

where $K_{L}$ is given by Eq. (6). We can rewrite Eq. (20) as

$$
\begin{aligned}
\frac{d \rho(t)}{d t}= & -i \lambda\left[A^{\dagger} A, \rho(t)\right]+K_{L}\left[2 A^{2} \rho(t) A^{\dagger 2}-A^{\dagger 2} A^{2} \rho(t)\right. \\
& \left.-\rho(t) A^{\dagger 2} A^{2}\right] .
\end{aligned}
$$

We notice that apart from the first term on the right-hand side in Eq. (21) this equation is exactly the same as Eq. (5). 
The first term arises because of the dynamic Stark shift that appears automatically once the three-level dynamics is described from first principles.

\section{PRODUCTION OF SCHRÖDINGER CAT STATES}

In this section we introduce the parametric pumping and determine the steady state of the quadratically pumped and damped cavity field for small Rabi angles of the damping atoms. The Hamiltonian that describes the parametric process may be written in the rotating-wave approximation as

$$
H_{G}=\hbar \omega A^{\dagger} A-\hbar\left(e^{-2 i \omega t} \xi_{G} A^{\dagger 2}+\xi_{G}^{*} e^{2 i \omega t} A^{2}\right),
$$

where $\omega$ is the frequency of the cavity field and $\xi_{G}$ is the parametric coupling constant. Our previous results in Sec. II are given in the interaction picture and so we perform a change of basis such that Eq. (22) becomes

$$
V_{G}=i \hbar K_{G}\left(A^{\dagger 2}-A^{2}\right) \text {, }
$$

where $\xi_{G}=i K_{G}$ and $\xi_{G}^{*}=-i K_{G}$. (This choice of $K_{G}$ is made to ensure slightly simpler equations for the steady state and the quantum jump simulations.) Thus, for the parametric process alone, we obtain the equation of motion for the density matrix in the interaction picture as

$$
\frac{d \rho(t)}{d t}=K_{G}\left[\left(A^{\dagger 2}-A^{2}\right), \rho(t)\right]
$$

Now, since we assume that the two-photon parametric pumping and two-photon absorption processes are independent we simply add the rates of change in the density matrix for the two processes. Therefore, taking into account Eqs. (21) and (24), we can write

$$
\begin{aligned}
\frac{d \rho(t)}{d t}= & -i \lambda\left[A^{\dagger} A, \rho(t)\right]+K_{L}\left[2 A^{2} \rho(t) A^{\dagger 2}-A^{\dagger 2} A^{2} \rho(t)\right. \\
& \left.-\rho(t) A^{\dagger 2} A^{2}\right]+K_{G}\left[\left(A^{\dagger 2}-A^{2}\right), \rho(t)\right] .
\end{aligned}
$$

Now, we again make use of Eq. (18) and

$$
\begin{aligned}
\frac{d \widetilde{\rho}(t)}{d t}= & K_{L}\left[2 \widetilde{A} \widetilde{A} \widetilde{\rho}(t) \widetilde{A}^{\dagger} \widetilde{A}^{\dagger}-\widetilde{A}^{\dagger} \widetilde{A^{\dagger}} \widetilde{A} \widetilde{A} \widetilde{\rho}(t)-\widetilde{\rho}(t) \widetilde{A}^{\dagger} \widetilde{A^{\dagger}} \widetilde{A} \widetilde{A}\right] \\
& +K_{G}\left[\left(\widetilde{A^{\dagger}} \widetilde{A}^{\dagger}-\widetilde{A} \widetilde{A}\right), \widetilde{\rho}(t)\right],
\end{aligned}
$$

where $\widetilde{A}=e^{-i A^{\dagger} A \lambda t} A e^{i A^{\dagger} A \lambda t}=e^{i \lambda t} A$ (in the following sections we drop the tilde in order to simplify notation).

The steady state of Eq. (26) can be obtained easily $[5,6]$, and if we write $\alpha=\sqrt{K_{G} / K_{L}}$, the steady-state solution is an even coherent state

$$
|\alpha\rangle_{e}=\left\{2\left[1+\exp \left(-2 \alpha^{2}\right)\right]\right\}^{-1 / 2}[|\alpha\rangle+|-\alpha\rangle]
$$

if the initial state of the field is comprised of even photon numbers only $[5,6]$ (such as the vacuum state). This is because the density matrix equation (26) consists entirely of two-photon processes and so for an initial state comprised of only even photon numbers the evolution of the density matrix will never produce any odd photon numbers. If the initial state is, for example, the one-photon Fock state, then the steady state of the field is the odd coherent state $[5,6]$

$$
|\alpha\rangle_{o}=\left\{2\left[1-\exp \left(-2 \alpha^{2}\right)\right]\right\}^{-1 / 2}[|\alpha\rangle-|-\alpha\rangle] .
$$

The two components of the even and odd coherent states $|\alpha\rangle$ and $|-\alpha\rangle$ can be quite distinct if the amplitude $\alpha$ has a length rather greater than unity. As a result, the combination of parametric pumping and two-photon dissipation (which, in this case, is provided by an atomic beam of three-level atoms) can be said to produce Schrödinger cat states $[5,6]$.

\section{MASTER EQUATION FOR RABI ANGLES OF ARBITRARY MAGNITUDE}

In this section we follow a more rigorous procedure than that in Sec. III in order to obtain the master equation describing the competition between the parametric pumping process and the two-photon absorption process we are considering here (a stream of three-level two-photon absorbing atoms). As we shall see, under certain conditions we can relax the assumption of small Rabi angles used in the preceding section. [The master equation obtained in this section reduces to Eq. (26) if we assume small Rabi angles.] The derivation below, besides being a generalization of the derivation of Eq. (26), will serve to shed some light on the system being studied here and on the approach proposed in Sec. V.

As we have seen, our system consists of a resonant cavity mode of the electromagnetic field pumped externally by a two-photon parametric process. A stream of three-level atoms enter the cavity in their lower level $|c\rangle$. These atoms play the role of the two-photon absorber; before they enter the cavity we assume that a regular stream of the atoms passes through an excitation region where the lower state $|c\rangle$ is created with a probability $p$ from some ground-state level below $|c\rangle$. (In the case of Rydberg atoms [9] $|a\rangle,|b\rangle$, and $|c\rangle$ will be highly excited Rydberg states lying well above the ground state.) We note that when $p$ is very small, the arrival statistics of atoms in the lower state $|c\rangle$ is Poissonian [17] (and see also the Poissonian limit of Refs. $[18,19])$. If the rate at which the atoms arrive at the excitation region is $R=1 / t_{\text {at }}$, then the average rate of injection of the atoms in the state $|c\rangle$ into the cavity is $r=p R$.

In order to derive a master equation for the field reduced density matrix, let us assume that during the short time that each atom spends in the cavity $t_{\text {int }}$, the change in the field reduced density matrix due to the parametric pumping is negligible. This is true if we assume that $K_{G} t_{\text {int }} \ll 1$.

The change in the field reduced density matrix due to the parametric pumping process only is

$$
\rho(t+\Delta t)=\mathcal{U}(\Delta t) \rho(t) \mathcal{U}^{\dagger}(\Delta t) \equiv M_{G}(\Delta t) \rho(t)
$$

where

$$
\mathcal{U}(\Delta t)=\exp \left(-\frac{i}{\hbar} V_{G} \Delta t\right)
$$

The part of the change in the field reduced density matrix due to only the interaction with one two-photon absorbing atom is given by 


$$
\rho\left(t+t_{\text {int }}\right)=\hat{S} \rho(t) \hat{S}^{\dagger}+\hat{C} \rho(t) \hat{C}^{\dagger} \equiv M_{L}\left(t_{\text {int }}\right) \rho(t),
$$

where

$$
\begin{gathered}
\hat{S}=A^{2} \frac{\sin [\sqrt{\hat{N}(\hat{N}-1)} \tau]}{\sqrt{\hat{N}(\hat{N}-1)}}, \\
\hat{C}=\cos [\sqrt{\hat{N}(\hat{N}-1)} \tau],
\end{gathered}
$$

and $\tau=\lambda t_{\text {int }}$ [see Eqs. (13)-(16)]. Therefore, we can write for the change in the field reduced density matrix after $K=R \Delta t$ atoms have passed through the cavity

$$
\begin{aligned}
\rho(t+\Delta t)= & {\left[(1-p) M_{G}\left(t_{\mathrm{at}}\right)+p M_{G}\left(t_{\mathrm{at}}\right) M_{L}\left(t_{\mathrm{int}}\right)\right]^{K} \rho(t) } \\
= & {\left[(1-p) M_{G}(p / r)+p M_{G}(p / r) M_{L}\left(t_{\mathrm{int}}\right)\right]^{r \Delta t / p} } \\
& \times \rho(t),
\end{aligned}
$$

where

$$
\begin{aligned}
M_{G}\left(t_{\text {at }}\right) \rho(t)= & \exp \left[-K_{G} \frac{p}{r}\left(A^{\dagger 2}-A^{2}\right)\right] \rho(t) \\
& \times \exp \left[K_{G} \frac{p}{r}\left(A^{\dagger 2}-A^{2}\right)\right]
\end{aligned}
$$

and we have used $t_{\mathrm{at}}=R^{-1}=p / r$. (Note the exchange of roles between pumping and damping compared to the conventional theory as in, e.g., $[18,19]$.) We show in Appendix $B$ that when we coarse grain the master equation we obtain, in the limit $p \rightarrow 0$,

$$
\frac{d \rho}{d t}=-\frac{i}{\hbar}\left[V_{G}, \rho(t)\right]+r\left[M_{L}-1\right] \rho(t),
$$

which is in form of the master equation for Poissonian pumping statistics [17] (see the Poissonian limit in Refs. $[18,19])$.

\section{QUANTUM JUMP APPROACH}

In this section we use the Monte Carlo wave-function methods $[14,15]$ to simulate the dissipative process for the large Rabi angle case. The quantum simulation describes the time evolution of a single realization subjected to quantum jumps when dissipative processes take place. We start from a "mesoscopic" point of view where the system is described by the coarse-grained master equation obtained in the preceding section.

We defined in Sec. IV the average rate of atomic injection $r=p R$. The probability of finding a single atom in an infinitesimal interval $[t, t+\delta t]$ is then given by $\delta P=r \delta t$. Let us take the master equation (36), but with $\Delta t$ replaced by the very small time interval $\delta t$ such that $r \delta t \ll 1$. [This differs from Eq. (B3) because we are now considering a small time step for the coarse-grained master equation.] We will see below that in this limit we can identify $r \delta t$ as the probability for a quantum jump to occur as a result of performing a selective measurement of the atomic state after the atom leaves the cavity. We now begin our derivation of a quantum simulation procedure by rewriting the master equation (36) in terms of the very small time step $\delta t$ :

$$
\rho(t+\delta t)=\rho(t)-\frac{i}{\hbar} r \delta t\left[\frac{V_{G}}{r}, \rho(t)\right]+r \delta t\left[M_{L}\left(t_{\mathrm{int}}\right)-1\right] \rho(t) .
$$

We assume Poissonian statistics for the incoming atoms and we rewrite here the expression for $M_{L}$,

$$
M_{L}\left(t_{\text {int }}\right) \rho(t)=\hat{S} \rho(t) \hat{S}^{\dagger}+\hat{C} \rho(t) \hat{C}^{\dagger},
$$

where $\hat{S}$ and $\hat{C}$ are given by Eqs. (32) and (33). For small Rabi angles it is easy to verify that we recover Eq. (5) from Eq. (38) (with $\dot{\rho} \rightarrow\left[M_{L}\left(t_{\text {int }}\right)-1\right] \rho(t) / t_{\text {int }}$ ). Now we notice that

$$
\hat{S}^{\dagger} \hat{S}+\hat{C}^{\dagger} \hat{C}=1
$$

and Eq. (37) can be cast in the form

$$
\begin{aligned}
\rho(t+\delta t)= & -\frac{i}{\hbar} r \delta t\left[\frac{V_{G}}{r}, \rho(t)\right]+\rho(t) \\
& +r \delta t\left[\hat{S} \rho(t) \hat{S}^{\dagger}-\frac{1}{2} \hat{S}^{\dagger} \hat{S} \rho(t)-\frac{1}{2} \rho(t) \hat{S}^{\dagger} \hat{S}\right] \\
& +r \delta t\left[\hat{C} \rho(t) \hat{C}^{\dagger}-\frac{1}{2} \hat{C}^{\dagger} \hat{C} \rho(t)-\frac{1}{2} \rho(t) \hat{C}^{\dagger} \hat{C}\right] .
\end{aligned}
$$

The dissipative part of this master equation is now in the Lindblad form [20] and so the whole master equation can be unraveled [14] into individual trajectories as shown below.

Following the state vector Monte Carlo method presented in Refs. [14,15], we define the state vector

$$
\left|\psi^{(1)}(t+\delta t)\right\rangle=\frac{e^{-(i / \hbar) r H_{\mathrm{eff}} \delta t}}{\sqrt{1-\delta P}}|\psi(t)\rangle
$$

where

$$
H_{\mathrm{eff}}=i \hbar \frac{K_{G}}{r}\left(A^{\dagger 2}-A^{2}\right)-i \frac{\hbar}{2}\left(\hat{S}^{\dagger} \hat{S}+\hat{C}^{\dagger} \hat{C}\right),
$$

and we further define the time evolved state vectors

$$
\begin{aligned}
\left|\psi^{(2)}(t+\delta t)\right\rangle & =\frac{\hat{C}}{\sqrt{\delta P_{C}}}|\psi(t)\rangle \sqrt{r \delta t}, \\
\left|\psi^{(3)}(t+\delta t)\right\rangle & =\frac{\hat{S}}{\sqrt{\delta P_{S}}}|\psi(t)\rangle \sqrt{r \delta t} .
\end{aligned}
$$

One of these state vectors will be chosen at every time step in the simulation. If we make use of Eqs. (41)-(44) we can show that, to first order in $\delta t$, the "jump probabilities" for the operators $\hat{S}$ and $\hat{C}$ satisfy

$$
\delta P_{S}+\delta P_{C}=\delta P=r \delta t .
$$

We now use Eqs. (41)-(44) again and define the relative probabilities 


$$
\begin{gathered}
\Pi_{S}=\frac{\delta P_{S}}{\delta P}=\left\langle\psi(t)\left|\hat{S}^{\dagger} \hat{S}\right| \psi(t)\right\rangle, \\
\Pi_{C}=\frac{\delta P_{C}}{\delta P}=\left\langle\psi(t)\left|\hat{C}^{\dagger} \hat{C}\right| \psi(t)\right\rangle .
\end{gathered}
$$

Using the above definitions we can show that the weighted random choice of the state vectors $\left|\psi^{(1-3)}\right\rangle$ is equivalent to the master equation (37). In order to do so, we first make some definitions. We define $e$ as the ensemble index and for the ensemble member $e, k_{e}=1,2,3$ depending on whether state $\left|\psi^{(1)}\right\rangle,\left|\psi^{(2)}\right\rangle$, or $\left|\psi^{(3)}\right\rangle$ is chosen in the simulations that will be discussed below. If the number of members of the ensemble is $J_{\text {ens }}$, then the ensemble-averaged density operator is

$$
\overline{\rho\left(t, J_{\mathrm{ens}}\right)}=\frac{1}{J_{\mathrm{ens}}} \sum_{e=1}^{J_{\mathrm{ens}}}\left|\psi^{\left(k_{e}\right)}(t)\right\rangle\left\langle\psi^{\left(k_{e}\right)}(t)\right|
$$

where $\left|\psi^{\left(k_{e}\right)}(t)\right\rangle\left\langle\psi^{\left(k_{e}\right)}(t)\right|$ is a conditioned density operator [14] and expression (47) is the average over the members of our representative ensemble at time $t$. Then, at time $t+\delta t$ the ensemble average gives us (in the limit $J_{\text {ens }} \rightarrow \infty$ )

$$
\begin{aligned}
\overline{\rho(t+\delta t)}= & (1-\delta P)\left|\psi^{(1)}(t+\delta t)\right\rangle\left\langle\psi^{(1)}(t+\delta t)\right| \\
& +\delta P\left[\Pi_{S}\left|\psi^{(2)}(t+\delta t)\right\rangle\left\langle\psi^{(2)}(t+\delta t)\right|\right. \\
& \left.+\Pi_{C}\left|\psi^{(3)}(t+\delta t)\right\rangle\left\langle\psi^{(3)}(t+\delta t)\right|\right] \\
= & |\psi(t)\rangle\langle\psi(t)|+r \delta t \\
& \times\left[\frac{K_{G}}{r}\left(A^{\dagger 2}-A^{2}\right),|\psi(t)\rangle\langle\psi(t)|\right]+r \delta t[\hat{S}|\psi(t)\rangle \\
& \left.\times\left\langle\psi(t)\left|\hat{S}^{\dagger}+\hat{C}\right| \psi(t)\right\rangle\left\langle\psi(t)\left|\hat{C}^{\dagger}-\right| \psi(t)\right\rangle\langle\psi(t)|\right] .
\end{aligned}
$$

Then we finally can write

$$
\frac{d \overline{\rho(t)}}{d t}=-\frac{i}{\hbar} r\left[\frac{V_{G}}{r}, \overline{\rho(t)}\right]+r\left[M_{L}\left(t_{\mathrm{int}}\right)-1\right] \overline{\rho(t)},
$$

which is equivalent to Eq. (37) if we write $\overline{\rho(t)}=\rho(t)$. In this way we have obtained the correct master equation that describes the competition between parametric pumping and two-photon absorption due to the three-level atoms that enter the cavity with a statistics characterized by the parameter $p$, where now the Rabi angles of the two-photon absorbing atoms can be large.

To perform the Monte Carlo wave function simulation, we choose a random number $\gamma$, which is uniformly distributed between 0 and 1 , and compare it with $\delta P$. If $\delta P$ is smaller than $\gamma$, no quantum jump is deemed to have occurred and the state of the system at $t+\delta t$ is given by Eq. (41). If $\delta P$ is larger than $\gamma$ a quantum jump occurs. Then, if $\delta P_{S}=\Pi_{S} \delta P \geqslant \gamma$, the jump will be an "S jump" and the state of the system at $t+\delta t$ will be given by Eq. (44). If $\delta P_{S}<\gamma$ the jump will be a " $C$ jump" and the state of the system will be given by Eq. (43).
In order to simulate the time evolution of the system we choose the values of $\delta P=r \delta t, \tau=\lambda t_{\text {int }}$, and $\alpha^{2}=K_{G} / K_{L}$. We then calculate $K_{L}=r \tau^{2} / 2$ and $K_{G}=\alpha^{2} K_{L}$ and at each time step we find the random number $\gamma$ and

$$
\begin{gathered}
\Pi_{S}=\sum_{N}|\langle N+2 \mid \psi(t)\rangle|^{2} \alpha_{N}, \\
\Pi_{C}=1-\Pi_{S},
\end{gathered}
$$

where $\alpha_{N}$ is given by Eq. (16). Then we write the state vectors (42)-(44) in the Fock basis and, taking into account Eqs. (41)-(44), we choose the state of the system according to the following rules. (a) If $\delta P<\gamma$,

$$
\begin{aligned}
\left\langle N \mid \psi^{(1)}(t+\delta t)\right\rangle= & \langle N \mid \psi(t)\rangle+\frac{K_{G} \delta t}{\sqrt{1-\delta P}}\{\sqrt{N(N-1)} \\
& \times\langle N-2 \mid \psi(t)\rangle-\sqrt{(N+1)(N+2)} \\
& \times\langle N+2 \mid \psi(t)\rangle\} .
\end{aligned}
$$

(b) If $\delta P \geqslant \gamma$ and (i) if $\delta P_{S}<\gamma$,

$$
\left\langle N \mid \psi^{(2)}(t+\delta t)\right\rangle=\frac{1}{\sqrt{\Pi_{C}}} \cos [\sqrt{(N)(N-1)} \tau]\langle N \mid \psi(t)\rangle,
$$

and (ii) if $\delta P_{S} \geqslant \gamma$,

$$
\left\langle N \mid \psi^{(3)}(t+\delta t)\right\rangle=\frac{1}{\sqrt{\Pi_{S}}} \sin [\sqrt{(N+1)(N+2)} \tau]\langle N+2 \mid \psi(t)\rangle
$$

where $K_{G} \delta t=\delta P \alpha^{2} \tau^{2} / 2$. This simulation, once averaged over many realizations, will then reproduce the results of the master equations (40) and (48).

We next need to calculate the behavior of observable quantities from these simulations. The ensemble-averaged density operator is

$$
\bar{\rho}\left(t, J_{\mathrm{ens}}\right)=\frac{1}{J_{\mathrm{ens}}} \sum_{e=1}^{J_{\mathrm{ens}}}\left|\psi^{\left(k_{e}\right)}(t)\right\rangle\left\langle\psi^{\left(k_{e}\right)}(t)\right| .
$$

From the above expression we can calculate the mean photon number

$$
\begin{aligned}
\langle N\rangle\left(t, J_{\text {ens }}\right) & =\operatorname{Tr}\left[\hat{N} \bar{\rho}\left(t, J_{\text {ens }}\right)\right] \\
& =\frac{1}{J_{\text {ens }}} \sum_{e=1}^{J_{\text {ens }}}\left\langle\psi^{\left(k_{e}\right)}(t)|\hat{N}| \psi^{\left(k_{e}\right)}(t)\right\rangle,
\end{aligned}
$$

the variance

$$
\begin{aligned}
\Sigma\left(t, J_{\text {ens }}\right)= & \left\langle\hat{N}^{2}\right\rangle\left(t, J_{\text {ens }}\right)-\langle\hat{N}\rangle^{2}\left(t, J_{\text {ens }}\right)=\operatorname{Tr}\left[\hat{N}^{2} \bar{\rho}\left(t, J_{\text {ens }}\right)\right] \\
& -\left\{\operatorname{Tr}\left[\hat{N} \bar{\rho}\left(t, J_{\text {ens }}\right)\right]\right\}^{2}
\end{aligned}
$$

and any other observable that we desire. 


\section{NUMERICAL RESULTS}

In this section we present the numerical results obtained following the procedure described in Sec. V. In Figs. 2(a)2(f) we show our numerical results for $\alpha^{2}=K_{G} / K_{L}=4$, $\delta P=r \delta t=10^{-2}, \tau=\lambda t_{\text {int }}=10^{-2}$, and $r=10^{2}$ for a cavity field starting from the vacuum. The ordinate axis $N_{\text {it }}$ represents the number of iterations of the scheme given by Eqs. (51)-(53). In Fig. 2(a) we show the mean photon number $\langle N\rangle$ and photon number variance $\Sigma=\left\langle N^{2}\right\rangle-\langle N\rangle^{2}$ for only one member of the ensemble. In this figure we can see jumps in $\langle N\rangle$ and $\Sigma$ that finally converge to a value of about 4.0, but with only small fluctuations around the final value. Although there are still some residual fluctuations, we notice that both mean photon number and photon number variance evolve together, experiencing the same small fluctuations for large enough times (for $N_{\text {it }}$ larger than $\approx 1.5 \times 10^{6}$ ). This confirms that the steady state is an even coherent state. Figure 2(b) shows the same results as those presented in Fig. 2(a); however, we have now superposed these with the history of the system time evolution (triangles). In this case the values 1,2 , and 3 are selected when the state vector $\left|\psi_{e}^{(1)}\right\rangle$, $\left|\psi_{e}^{(2)}\right\rangle$, or $\left|\psi_{e}^{(3)}\right\rangle$ is chosen, respectively [cf. Eqs. (41)-(44)]. The frequent selection of $\left|\psi_{e}^{(1)}\right\rangle$ and $\left|\psi_{e}^{(2)}\right\rangle$ results in the solid "bars" across the figure; however, the selection of $\left|\psi_{e}^{(3)}\right\rangle$ can be clearly seen. As we see, the more drastic jumps happen when the stochastic wave function $\left|\psi_{e}^{(3)}\right\rangle$ is chosen. The reason for these jumps being more accentuated is that the atoms enter the cavity in the lower state and as the selection of state $\left|\psi_{e}^{(3)}\right\rangle$ corresponds to the detection of the atoms in the upper state, we observe a more drastic change in the state of the system whenever $\left|\psi_{e}^{(3)}\right\rangle$ is selected. In Figs. 2(c)-2(f) we show our results for 1 (dotted curve), 10 (dash-dotted curve), and 30 (full curve) samples of the system representative ensemble. In all the graphs we see the jumps wash out when we take more and more members of the ensemble into account. In Fig. 2(f) we plot the cavity field quadrature variance $\Delta X_{2}=\sqrt{\left\langle X_{2}^{2}\right\rangle-\left\langle X_{2}\right\rangle^{2}}$ and note this result agrees with that obtained in Ref. [21]. Figure 2(e) shows the quadrature fluctuations $\Delta X_{1}=\sqrt{\left\langle X_{1}^{2}\right\rangle-\left\langle X_{1}\right\rangle^{2}}$. In Fig. 2(g) we depict an indication of the cavity field purity $\operatorname{Tr} \rho^{2}$. As we see, the field evolves first to a statistical mixture and then, in the steady state, becomes a pure state [an even coherent state which is close to Eq. (27) because of the small value of $\tau$ ]. In Fig. 3 we show a three-dimensional plot of the Wigner function for the same values of the parameters as in Fig. 2 and for $N_{\mathrm{it}}=2.0 \times 10^{6}$ corresponding to the steady state in Fig. 2 .
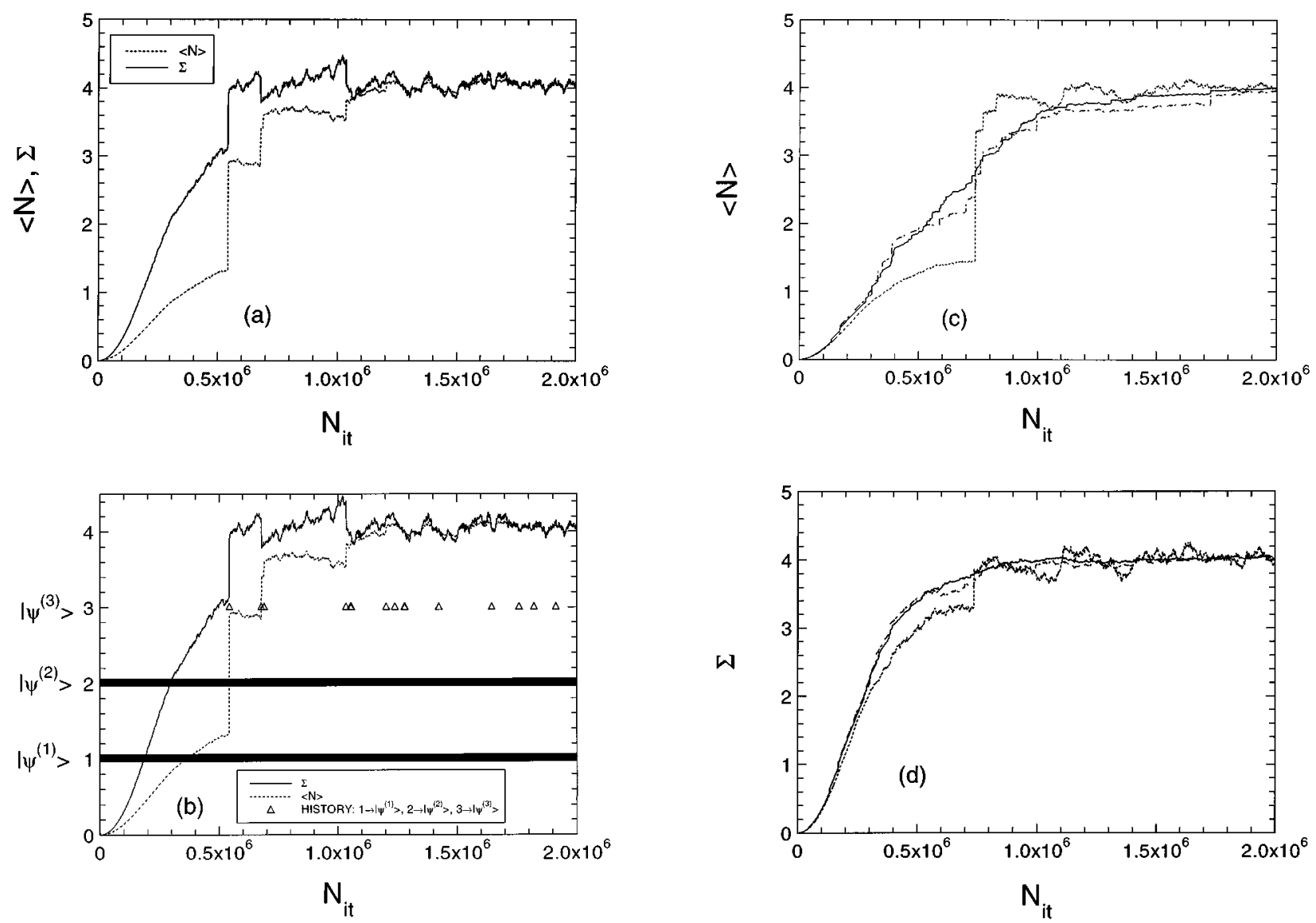

FIG. 2. Our numerical results for $\alpha^{2}=4, \delta P=10^{-2}, \tau=10^{-2}$, and $r=10^{2}$ starting from the vacuum. These results were calculated using the quantum-jump approach of Sec. V. The ordinate axis $N_{\text {it }}$ represents the number of interactions of the scheme shown by Eqs. (51)-(53). We have results for a single sample in (a) and (b) and show (a) the mean photon number $\langle N\rangle$ and variance $\Sigma=\left\langle N^{2}\right\rangle-\langle N\rangle^{2}$ and (b) the locations of the jumps into the state $\left|\psi^{(3)}\right\rangle$ are indicated with the triangles superimposed on the results of (a). In (c) -(f) we show our results for 1 (dotted curve), 10 (dash-dotted curve), and 30 (full curve) samples: (c) mean photon number $\langle N\rangle$, (d) photon number variance $\Sigma=\left\langle N^{2}\right\rangle-\langle N\rangle^{2}$, (e) variance $\Delta X_{1}$, and (f) variance $\Delta X_{2}$. In (g) we show the purity of the state $\operatorname{Tr} \rho^{2}$ with 30 samples. 

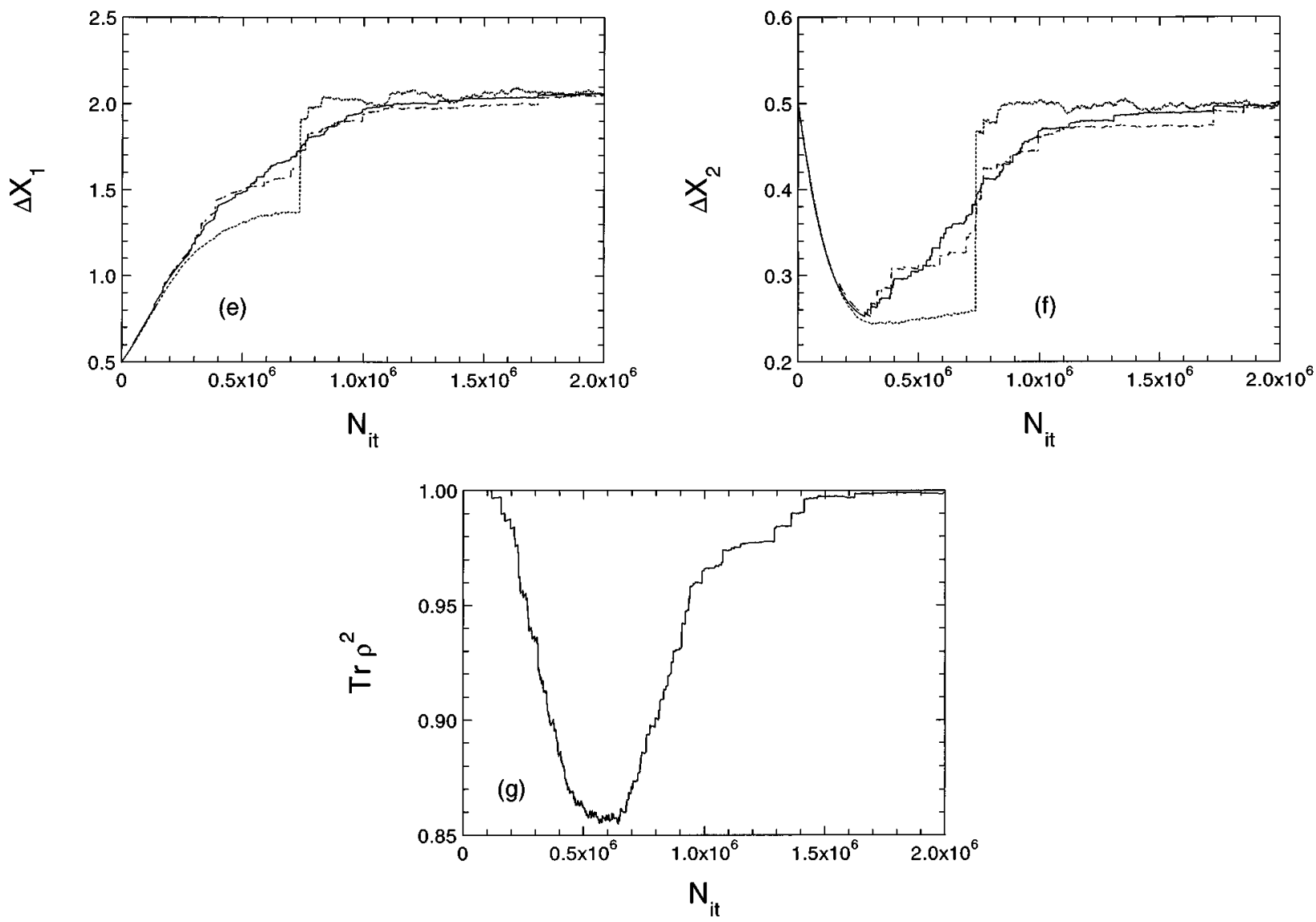

FIG. 2. (Continued).

The interference fringes in the Wigner function indicate the coherence between the two components of even coherent state.

In Figs. 4(a)-4(d) we present results for $\alpha^{2}=4$,

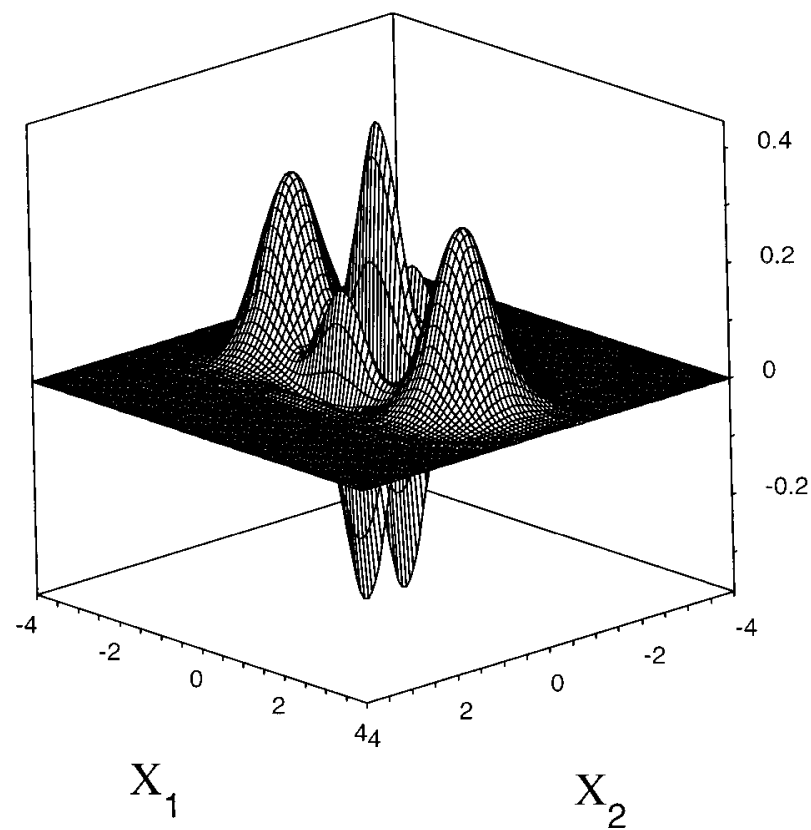

FIG. 3. Three-dimensional plot of the Wigner function for the same values of the parameters as in Fig. 2 and for $N_{\mathrm{it}}=2 \times 10^{6}$. $\delta P=10^{-2}, \tau=10^{-2}$, and $r=10^{2}$, but now with the field in the state $[\cos (\varphi / 2)|0\rangle+\sin (\varphi / 2)|1\rangle]$ for $\varphi=\pi / 4$. This admits odd Fock states as well as even Fock states. As we see in Fig. 4(d), the field steady state generated from this seed state is no longer a pure state. In Figs. 5(a)-5(d) we show our results for the same parameters as in Figs. 4(a)-4(d), but for $\varphi=\pi / 2$. In Fig. 5(d) we see that the deviation from a pure state is larger in this last case. This is because the initial state contains an equal superposition of even and odd Fock states (unlike Fig. 4 where the even states dominate) and this leads to a balanced statistical mixture of the even and odd coherent states.

In Figs. 6(a)-6(f) we increase the Rabi angle of the absorbing atoms and present our numerical results for $\alpha^{2}=4$, $\delta P=10^{-2}, \tau=10^{-1}$, and $r=10^{2}$ calculated by the quantumjump approach. Figures 6(a) and 6(b) show, respectively, $\langle N\rangle$ and $\Sigma$ for only one member of the ensemble. In Figs. 6(c) and 6(d) we employ the same parameters as in Figs. 6(a) and 6(b) but we show the results from an average over 100 samples. If we compare these results with those shown in Figs. 2(c) and 2(d) we see that the fluctuations in $\langle N\rangle$ and $\Sigma$ in the steady state have increased and also that the steady state is reached quickly. This happens because in this case the coupling constants of the three-level atoms are larger, which corresponds to larger Rabi angles such that these twophoton absorbing atoms are less efficient as pure absorbers and allow the photon distribution to be pushed towards larger photon numbers due to the parametric pumping process. In 

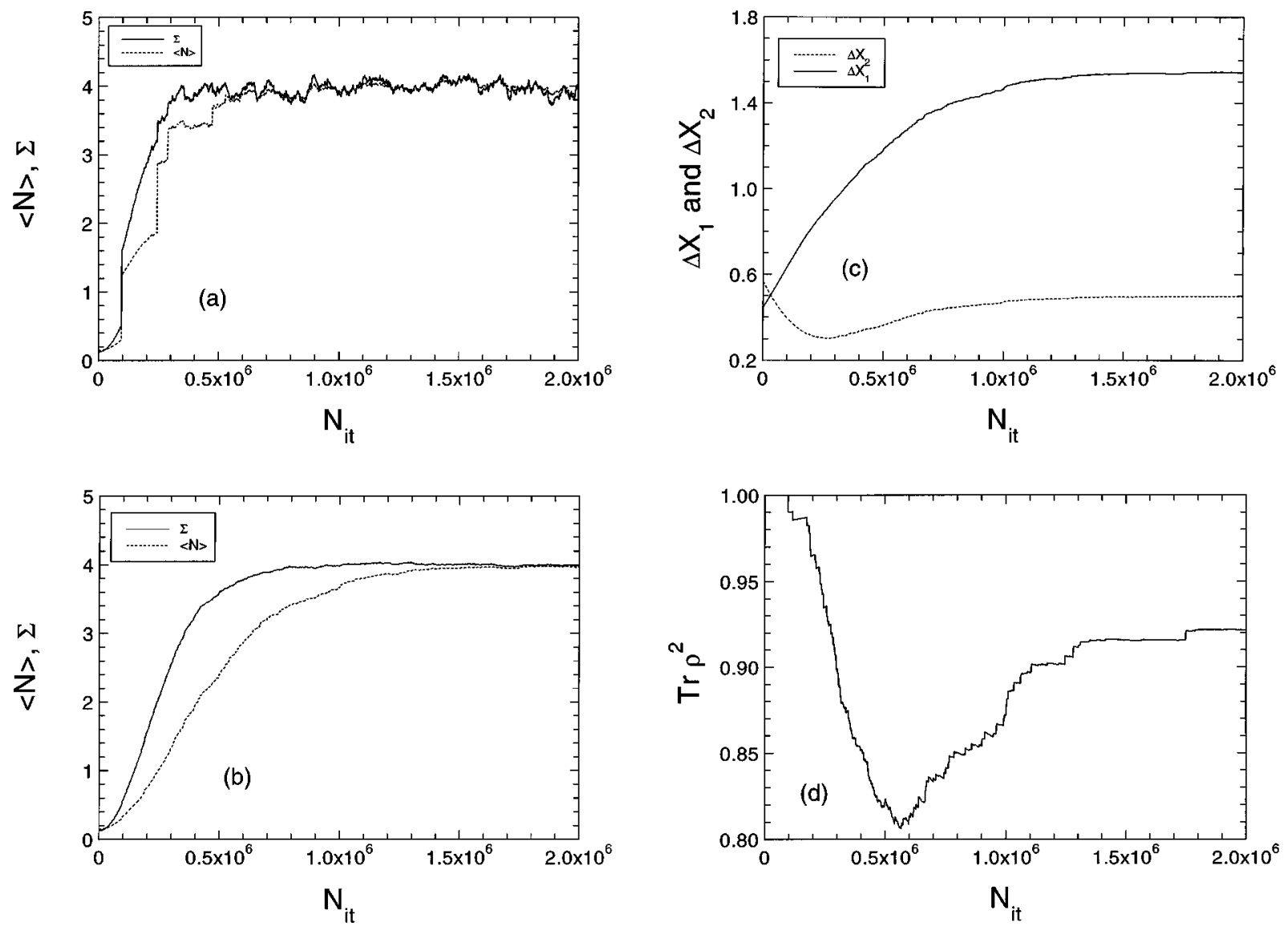

FIG. 4. Results for $\alpha^{2}=4, \quad \delta P=10^{-2}, \quad \tau=10^{-2}$, and $r=10^{2}$ with 100 samples. The initial state of the field is $\cos (\varphi / 2)|0\rangle+\sin (\varphi / 2)|1\rangle$ for $\varphi=\pi / 4$. We show (a) and (b) the mean photon number $\langle N\rangle$ and variance $\Sigma=\left\langle N^{2}\right\rangle-\langle N\rangle^{2}$ for 1 and 100 samples, respectively, (c) variances $\Delta X_{1}$ and $\Delta X_{2}$ (with 100 samples), and (d) the purity of the state $\operatorname{Tr} \rho^{2}$ (with 100 samples).

Fig. 6(f) we see that the steady state so obtained is not a pure state.

In the previous figures we saw that the dynamics of the system is strongly affected by a change in the coupling constant of the two-photon absorbing atoms. In order to demonstrate this feature of the system more clearly, we depict in Figs. 7(a)-7(e) our results for $\langle N\rangle, \Sigma, \Delta X_{1}, \Delta X_{2}$, and $\operatorname{Tr} \rho^{2}$ for $\tau=1 \times 10^{-2}, 2.5 \times 10^{-2}$, and $5 \times 10^{-2}$. As we see in these figures, when we increase the value of $\tau$, we reach the steady state more quickly. We notice also that comparing the results for $\tau=1 \times 10^{-2}$ and $2.5 \times 10^{-2}$, in the steady state, the deviation from a pure state of the system for this last case is not very large and the system reaches the steady state much more quickly than in the previous case.

\section{CONCLUSION}

We began this article with a simple heuristic derivation of the small Rabi angle master equation (5) describing a process of cavity field decay taking place two photons at a time. We then justified this with a more rigorous model based on the idea of a stream of three-level atoms passing through a cavity. The atoms act as an absorber of photons from the cavity field because the atoms all enter in their ground state. They act as two-photon absorbers because the cavity-atom interaction is at two-photon resonance. In the weak-coupling limit (small Rabi angles) we derived the master equation (21) to describe two-photon absorption of the cavity field. In this limit we have seen that if we parametrically pump the cavity field the steady state becomes an even coherent state (when the cavity is initially empty).

Our principle goal has been to develop a master equation to describe the absorption process when the interaction between the absorbing atoms and the cavity is weak or strong. By assuming Poissonian statistics for the incoming absorbing atoms we find the master equation (36) where the decay of the field is seen to be controlled by two operators $\hat{S}$ and $\hat{C}$ [Eqs. (32) and (33)], which depend nonlinearly on the interaction time $t_{\text {int }}$. The master equation could be solved directly by numerical methods, but we have chosen to explore the possibility of a state vector Monte Carlo simulation [Eqs. (51)-(53)]. This is possible because the master equation (36) [and the decay part $M_{L}-1$; see Eq. (31)] can be written in the Lindblad form [see Eq. (40)] allowing an unraveling into three processes: an $S$ jump, a $C$ jump, and no jump. The simulation of the master equation using the stochastic trajectories of an ensemble of state vectors has the advantage of using less computer memory than a direct integration of the density matrix.

The numerical results have utilized the quantum-jump simulations of Sec. V. For weak interactions (small Rabi angle) we have shown that we obtain the expected steadystate limit (starting with an unexcited cavity state): a pure even coherent state as in Eq. (27). Such a cavity field state is 

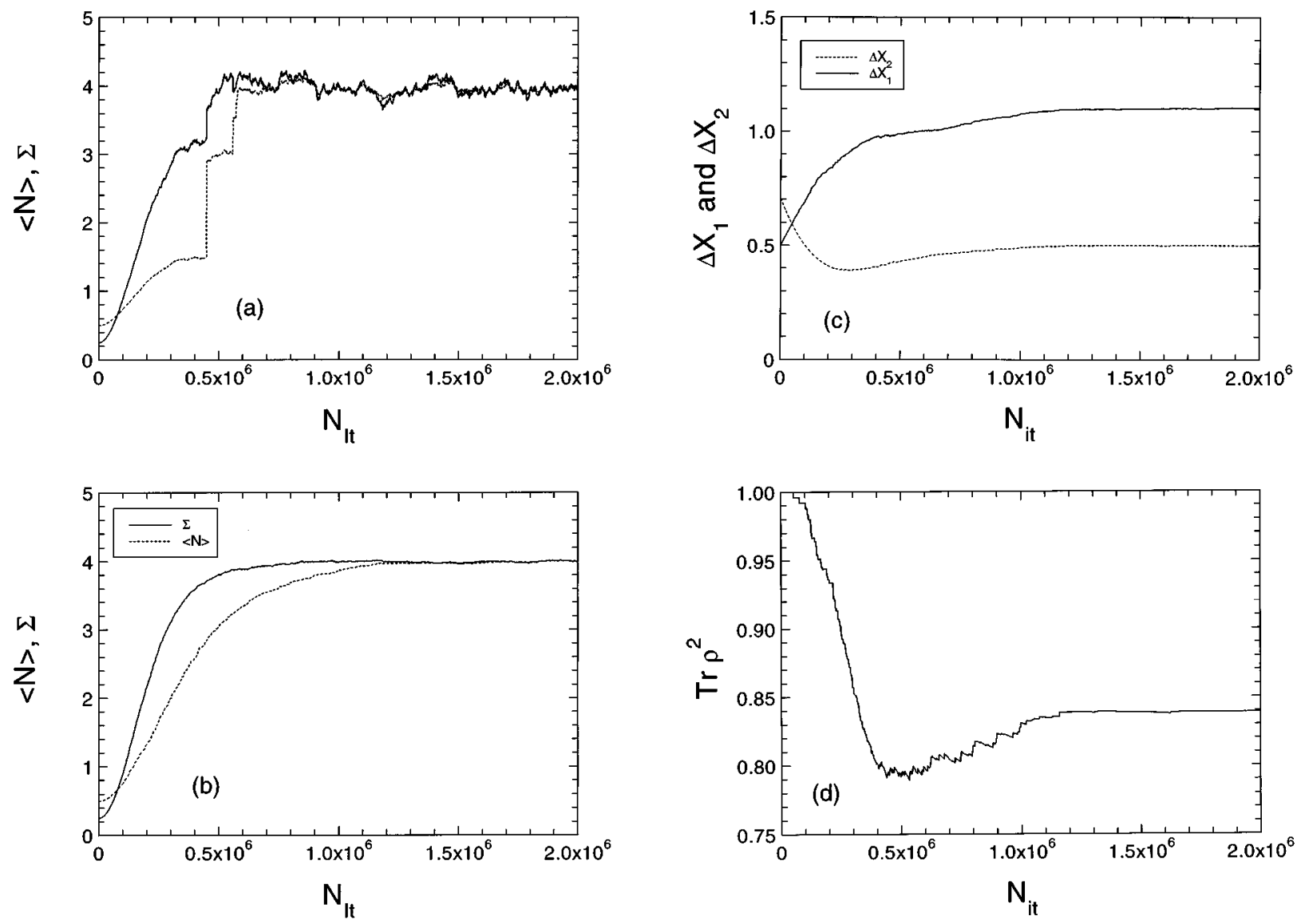

FIG. 5. Results for the same parameters as in Figs. 4(a)-4(d), but for $\varphi=\pi / 2$. We show (a) and (b) the mean photon number $\langle N\rangle$ and variance $\Sigma=\left\langle N^{2}\right\rangle-\langle N\rangle^{2}$ for 1 and 100 samples, respectively, (c) variances $\Delta X_{1}$ and $\Delta X_{2}$ (with 100 samples), and (d) the purity of the state $\operatorname{Tr} \rho^{2}$ (with 100 samples).

a microscopic superposition of two different (nonoverlapping) coherent states that is generated here by the interaction of the parametric pumping and the absorbing atoms with the cavity. However, we have seen that when the initial cavity state is changed to a superposition of even and odd Fock states we lose the purity of the steady state. Likewise, as the interaction time of the absorbing atoms is increased (increasing the Rabi angle of the interaction) the purity of the final state decreases.

\section{ACKNOWLEDGMENTS}

This work was supported by Conselho Nacional de Desenvolvimento Científico e Tecnológico, the European Community, and the United Kingdom Engineering and Physical Sciences Research Council.

\section{APPENDIX A: SOLUTION OF THE SYSTEM OF DIFFERENTIAL EQUATIONS (10)}

If we define

$$
\begin{gathered}
\zeta_{1}=i g_{a b} \sqrt{N+1}, \quad \zeta_{1}=i g_{b c} \sqrt{N+2}, \\
\mu_{1}=i \Delta_{1}, \quad \mu_{2}=i \Delta_{2},
\end{gathered}
$$

the system of differential equations (10) can be written as

$$
\begin{gathered}
e^{-\mu_{1} \tau} \dot{C}_{a, N}(\tau)=-\zeta_{1} C_{b, N+1}(\tau), \\
e^{\mu_{2} \tau} \dot{C}_{c, N+2}(\tau)=-\zeta_{2} C_{b, N+1}(\tau), \\
\dot{C}_{b, N+1}(\tau)=-\zeta_{1} e^{-\mu_{1} \tau} C_{a, N}(\tau)-\zeta_{2} e^{\mu_{2} \tau} C_{c, N+2}(\tau) .
\end{gathered}
$$

Now, if we use a Laplace transformation, the system of differential equations (A2) reduces to the set of algebraic equations

$$
\begin{gathered}
\left(z+\mu_{1}\right) \widetilde{C}_{a, N}\left(z+\mu_{1}\right)-C_{a, N}\left(t_{i}\right)=-\zeta_{1} \widetilde{C}_{b, N+1}(z) \\
\left(z-\mu_{2}\right) \widetilde{C}_{c, N+2}\left(z-\mu_{2}\right)-C_{c, N+2}\left(t_{i}\right)=-\zeta_{2} \widetilde{C}_{b, N+1}(z), \\
z \widetilde{C}_{b, N+1}(z)-C_{b, N+1}\left(t_{i}\right)=-\zeta_{1} \widetilde{C}_{a, N}\left(z+\mu_{1}\right) \\
-\zeta_{2} \widetilde{C}_{c, N+2}\left(z-\mu_{2}\right) .
\end{gathered}
$$

Solving Eq. (A3) for $\widetilde{C}_{b, N+1}(z)$ we find 

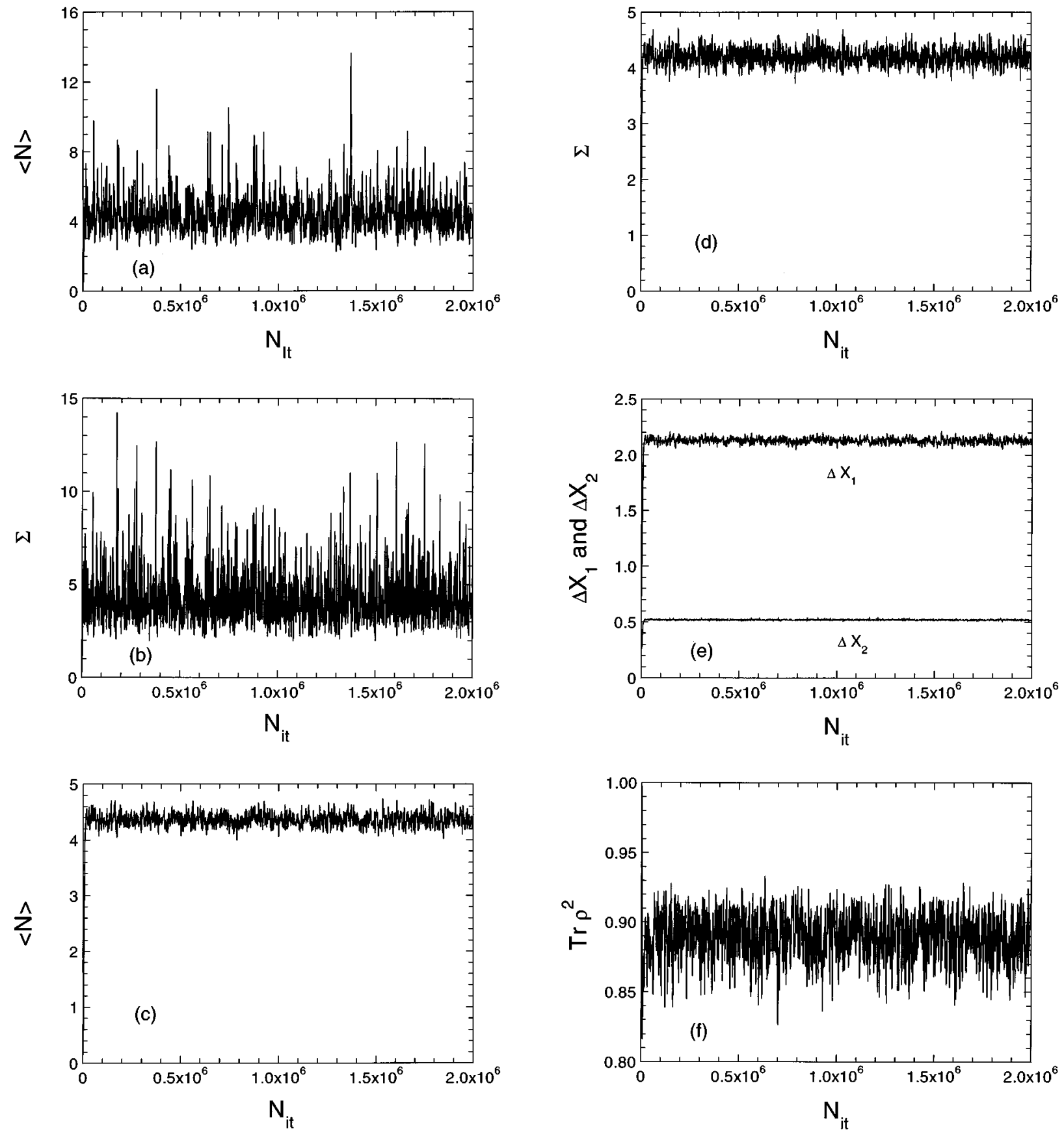

FIG. 6. Results for $\alpha^{2}=4, \delta P=10^{-2}, \tau=10^{-1}$, and $r=10^{2}$ calculated by the quantum-jump approach. (a) and (b), respectively, show $\langle N\rangle$ and $\Sigma$ for only one member of the ensemble. In (c) and (d) we show $\langle N\rangle$ and $\Sigma$ for 100 samples. In (e) we show the variances $\Delta X_{1}$ and $\Delta X_{2}$ (with 100 samples). In (f) we display the purity of the state $\operatorname{Tr} \rho^{2}$ (with 100 samples).

$$
\widetilde{C}_{b, N+1}(z)=\frac{\left(z+\mu_{1}\right)\left(z-\mu_{2}\right) C_{b, N+1}\left(t_{i}\right)-\zeta_{1}\left(z-\mu_{2}\right) C_{a, N}\left(t_{i}\right)-\zeta_{2}\left(z+\mu_{1}\right) C_{c, N+2}\left(t_{i}\right)}{z\left(z+\mu_{1}\right)\left(z-\mu_{2}\right)-\zeta_{1}^{2}\left(z-\mu_{2}\right)-\zeta_{2}^{2}\left(z+\mu_{1}\right)}
$$

and for the case in which $C_{b, N+1}\left(t_{i}\right)=C_{a, N}\left(t_{i}\right)=0$, Eq. (A4) simplifies to

$$
\widetilde{C}_{b, N+1}(z)=\frac{-C_{c, N+2}\left(t_{i}\right) \zeta_{2}\left(z+\mu_{1}\right)}{z\left(z+\mu_{1}\right)\left(z-\mu_{2}\right)-\zeta_{1}^{2}\left(z-\mu_{2}\right)-\zeta_{2}^{2}\left(z+\mu_{1}\right)}
$$

If $z_{1, N}, z_{2, N}$, and $z_{3, N}$ are the roots of the denominator of Eq. (A5) and we use the inverse Laplace transformation, the solution of Eq. (A2) can be written 

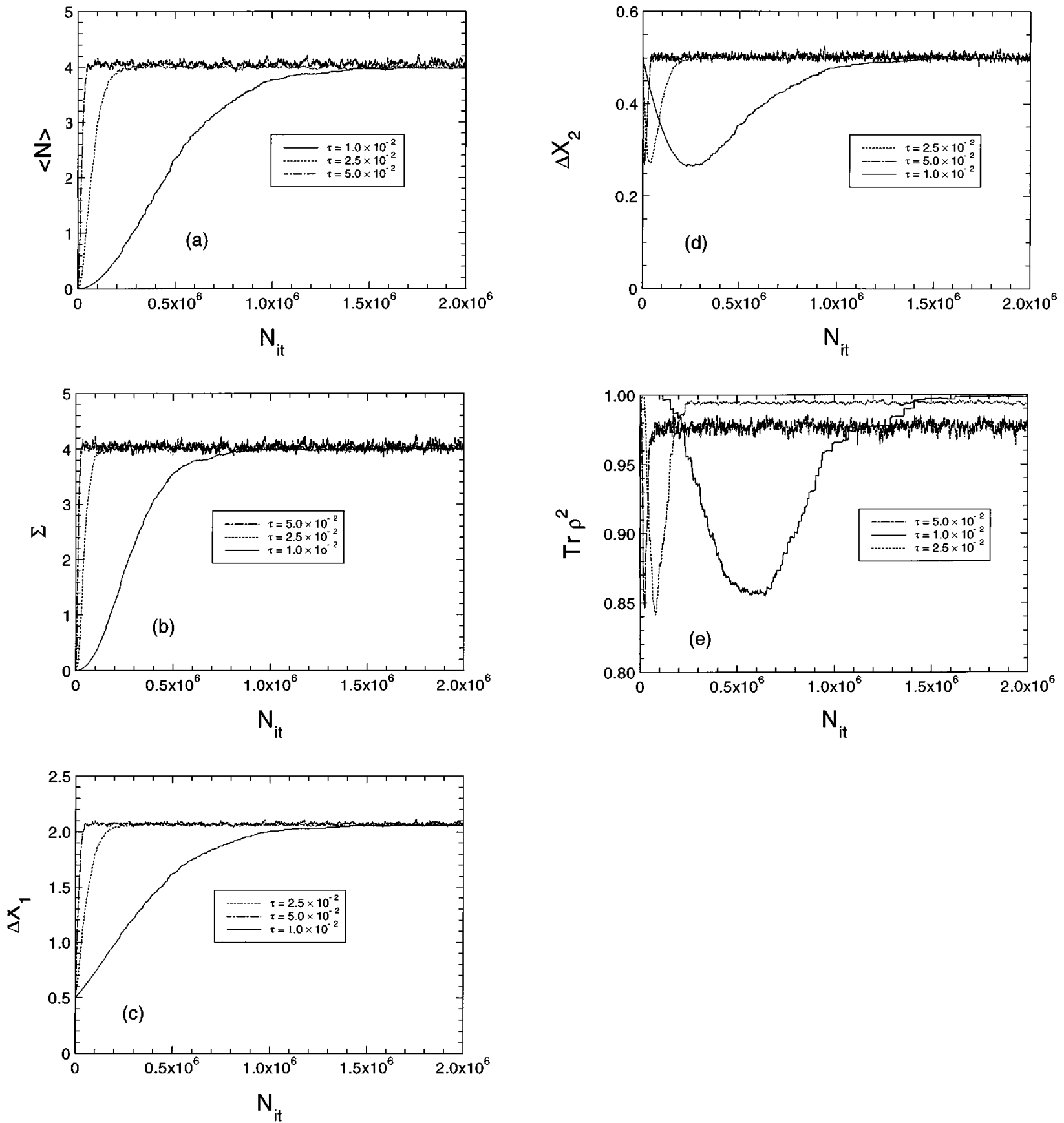

FIG. 7. (a) $\langle N\rangle$, (b) $\Sigma$, (c) $\Delta X_{1}$, (d) $\Delta X_{2}$, and (e) $\operatorname{Tr} \rho^{2}$ for $\tau=1 \times 10^{-2}, 2.5 \times 10^{-2}$, and $5 \times 10^{-2}$ and other parameters as in Fig. 6 (with 100 samples).

$$
\begin{gathered}
C_{a, N}\left(t_{i}+\tau\right)=C_{c, N+2}\left(t_{i}\right) \zeta_{1} \zeta_{2}\left\{\frac{e^{\left(z_{1, N}+\mu_{1}\right) \tau}}{\left(z_{1, N}-z_{2, N}\right)\left(z_{1, N}-z_{3, N}\right)}-\frac{e^{\left(z_{2, N}+\mu_{1}\right) \tau}}{\left(z_{1, N}-z_{2, N}\right)\left(z_{2, N}-z_{3, N}\right)}+\frac{e^{\left(z_{1, N}+\mu_{1}\right) \tau}}{\left(z_{2, N}-z_{3, N}\right)\left(z_{1, N}-z_{3, N}\right)}\right\}, \\
C_{b, N+1}\left(t_{i}+\tau\right)=-C_{c, N+2}\left(t_{i}\right) \zeta_{2}\left\{\frac{\left(z_{1, N}+\mu_{1}\right) e^{z_{1, N} \tau}}{\left(z_{1, N}-z_{2, N}\right)\left(z_{1, N}-z_{3, N}\right)}-\frac{\left(z_{2, N}+\mu_{1}\right) e^{z_{2, N} \tau}}{\left(z_{1, N}-z_{2, N}\right)\left(z_{2, N}-z_{3, N}\right)}+\frac{\left(z_{3, N}+\mu_{1}\right) e^{z_{1, N} \tau}}{\left(z_{2, N}-z_{3, N}\right)\left(z_{1, N}-z_{3, N}\right)}\right\}, \\
C_{c, N+2}\left(t_{i}+\tau\right)=C_{c, N+2}\left(t_{i}\right) \zeta_{2}^{2}\left\{\frac{\left(z_{1, N}+\mu_{1}\right) e^{\left(z_{1, N}+\mu_{1}\right) \tau}}{\left(z_{1, N}-z_{2, N}\right)\left(z_{1, N}-z_{3, N}\right)\left(z_{1, N}-\mu_{2}\right)}-\frac{\left(z_{2, N}+\mu_{1}\right) e^{\left(z_{2, N}+\mu_{1}\right) \tau}}{\left(z_{1, N}-z_{2, N}\right)\left(z_{2, N}-z_{3, N}\right)\left(z_{2, N}-\mu_{2}\right)}\right. \\
\left.+\frac{\left(z_{3, N}+\mu_{1}\right) e^{\left(z_{1, N}+\mu_{1}\right) \tau}}{\left(z_{2, N}-z_{3, N}\right)\left(z_{1, N}-z_{3, N}\right)\left(z_{3, N}-\mu_{2}\right)}\right\}
\end{gathered}
$$


Now we assume

$$
\frac{g_{a b}}{g_{b c}}=\frac{g_{a b}}{g}=1+\epsilon^{2}
$$

and

$$
\frac{\Delta_{1}}{\Delta_{2}}=\frac{\Delta}{\Delta_{2}}=-1+\epsilon^{2},
$$

where $\epsilon=g / \Delta$. In order that the two-photon transitions are dominant, one-photon transitions must be suppressed and therefore the detuning $\Delta$ has to be large compared to the coupling constants between the energy levels. We will study the cases in which

$$
g_{a b}, g_{b c} \ll \Delta .
$$

Therefore, $\epsilon \ll 1$ and

$$
\frac{g^{2}}{\Delta} \ll \Delta
$$

There is no need to solve the cubic equation in the denominator of Eq. (A5) exactly. We can find the roots $z_{1, N}, z_{2, N}$, and $z_{3, N}$ perturbatively under the assumptions (A6)-(A10).

We have to find the roots of the denominator of Eq. (A4), i.e., the roots of

$$
f(z)=z\left(z+\mu_{1}\right)\left(z-\mu_{2}\right)-\xi_{1}^{2}\left(z-\mu_{2}\right)-\xi_{2}^{2}\left(z+\mu_{1}\right),
$$

which we will call $z_{1}, z_{2}$, and $z_{3}$. Let us define

$$
r=\frac{\Delta_{2}}{\Delta_{1}}, \quad q=\frac{g_{a b}}{g_{b c}}, \quad p=q r=\frac{g_{a b} / \Delta_{1}}{g_{b c} / \Delta_{2}},
$$

where $\Delta_{1}=\Delta, g_{b c}=g$, and $|r|,|p|$, and $|q|$ are of the order of 1. Therefore, $\Delta_{2}=r \Delta$ and $g_{a b}=q g$. We define $x=z / \Delta$ and $\epsilon=g / \Delta$, and then Eq. (A11) can written as

$$
f(x)=x^{3}+i s x^{2}+\left[r-\phi \epsilon^{2}\right] x+i a_{2} \epsilon^{2},
$$

where

$$
\phi=-\left[q^{2}(N+1)-(N+2)\right]
$$

and

$$
a_{2}=-\left[r q^{2}(n+1)-(n+2)\right] .
$$

The solution of Eq. (A13) can be obtained more easily if we write

$$
f(x)=\left(x-x_{1}\right)\left(x^{2}+\alpha x+\beta\right),
$$

where

$$
\alpha=i s+x_{1}
$$

and

$$
\beta=\alpha x_{1}+r-\phi \epsilon^{2}
$$

If we obtain $x_{1}$ the other two roots are

$$
x_{2}=\frac{1}{2}\left[-\alpha+r_{N}\right], x_{3}=\frac{1}{2}\left[-\alpha-r_{N}\right] \text {, }
$$

where

$$
r_{N}=\sqrt{\alpha^{2}-4 \beta}
$$

In order to obtain $x_{1}$ we write

$$
x_{1}=c_{0}+c_{1} \epsilon+c_{2} \epsilon^{2}+c_{3} \epsilon^{3}+c_{4} \epsilon^{4} \text {. }
$$

If we substitute Eq. (A21) in Eq. (A13) and collect terms of order $\epsilon$, then to satisfy $f\left(x_{1}\right)=0$ we choose $c_{0}=0$. It follows that $c_{1}=c_{3}=0$,

$$
c_{2}=-i a_{2} / r
$$

and

$$
c_{4}=i\left[\frac{a_{2}\left(a_{2} s-\phi r\right)}{r^{3}}\right] .
$$

Substituting Eqs. (A17) and (A18) in Eq. (A20) we obtain

$$
\begin{aligned}
r_{N}^{2}= & \frac{1}{r^{3}}\left(N^{2} \epsilon^{4}\left(q^{2} r-1\right)\left[q^{2} r^{2}(2 r+1)-(r+2)\right]\right. \\
& +2 N \epsilon^{2}\left\{\epsilon^{2}\left[q^{4} r^{3}(2 r+1)-3 q^{2} r\left(r^{2}+r+1\right)+2(r+2)\right]\right. \\
& \left.-r^{2}(r+1)\left(q^{2} r+1\right)\right\}+\epsilon^{4}\left(q^{2} r-2\right)\left[q^{2} r^{2}(2 r+1)\right. \\
& \left.-(2 r+2)]-2 r^{2} \epsilon^{2}(r+1)\left(q^{2} r+2\right)-r^{3}\left(r^{2}+r+1\right)\right) .
\end{aligned}
$$

If we choose

$$
r=-1, \quad q=-1
$$

and $p=-1$. Substituting Eq. (A25) in Eq. (A24), we obtain

$$
r_{N}^{2}=-\left(\Omega_{N} / \Delta\right)^{2}
$$

where

$$
\Omega_{N}=(2 N+3) \frac{g^{2}}{\Delta} .
$$

If we choose

$$
r=-1+\epsilon^{2}, \quad q=-1+\epsilon^{2}
$$

and $p \approx-1$. Substituting Eq. (A28) in Eq. (A24), we obtain

$$
r_{N}^{2}=-\left(\frac{\Theta_{N}}{\Delta}\right)^{2}
$$

where

$$
\Theta_{N}=2 \sqrt{(N+1)(N+2)} \frac{g^{2}}{\Delta} .
$$




$$
\begin{gathered}
z_{1, N}=i \Omega_{N}, \\
z_{2, N}=-\frac{i}{2}\left\{\Delta-\frac{g^{2}}{\Delta}+\Omega_{N}+\Theta_{N}\right\}, \\
z_{2, N}=-\frac{i}{2}\left\{\Delta-\frac{g^{2}}{\Delta}+\Omega_{N}-\Theta_{N}\right\},
\end{gathered}
$$

or

$$
\begin{gathered}
z_{1}=i \Omega_{N}, \\
z_{2}=-i\left\{\Delta-\frac{g^{2}}{2 \Delta}\left[1-(\sqrt{N+1}-\sqrt{N+2})^{2}\right]\right\}, \\
z_{3}=-i\left\{\Delta-\frac{g^{2}}{2 \Delta}\left[1-(\sqrt{N+1}+\sqrt{N+2})^{2}\right]\right\} .
\end{gathered}
$$

Then the approximate expressions for Eq. (A6) are

$$
\begin{gathered}
C_{a, N}\left(t_{i}+\tau\right) \approx i e^{-i\left(g^{2} / \Delta\right)(\tau / 2)} C_{c, N+2}\left(t_{i}\right) e^{-i \Omega_{N}(\tau / 2)} \sin \left(\Theta_{N} \frac{\tau}{2}\right), \\
C_{b, N+1}\left(t_{i}+\tau\right) \approx 0, \\
C_{c, N+2}\left(t_{i}+\tau\right) \approx C_{c, N+2}\left(t_{i}\right) e^{-i \Omega_{N}(\tau / 2)} \cos \left(\Theta_{N} \frac{\tau}{2}\right) .
\end{gathered}
$$

Finally, let us assume that $g_{a b}$ and $g_{b c}=g$ are fixed and $p=1$. Then $r=1 / q=g_{b c} / g_{a b}=g / g_{a b}$ is fixed. Therefore, for $\Delta_{1}=\Delta$ we have

$$
\frac{g_{b c}}{g_{a b}}=\frac{g}{g_{a b}}=-1+\left(\frac{g}{\Delta}\right)^{2} .
$$

From Eq. (A34) we can obtain

$$
\Delta=g \sqrt{\frac{g_{a b}}{g-g_{a b}}}
$$

and $\Delta_{2}=r \Delta=\left(g / g_{a b}\right) \Delta$.

\section{APPENDIX B: DERIVATION OF THE MASTER EQUATION (36)}

We start with the short time evolution of the master equation (34),

$$
\begin{aligned}
\rho(t+\Delta t)= & {\left[(1-p) M_{G}\left(t_{\mathrm{at}}\right)+p M_{G}\left(t_{\mathrm{at}}\right) M_{L}\left(t_{\mathrm{int}}\right)\right]^{K} \rho(t) } \\
= & {\left[(1-p) M_{G}(p / r)+p M_{G}(p / r) M_{L}\left(t_{\mathrm{int}}\right)\right]^{r \Delta t / p} } \\
& \times \rho(t),
\end{aligned}
$$

where $M_{G}\left(t_{\text {at }}\right)$ is given by Eq. (35). Then, in order to write a differential equation for $\rho(t)$ we use $[18,19]$

$\rho(t+\Delta t)=\exp \left(\frac{r}{p} \Delta t \ln \left\{M_{G}\left[1+p\left(M_{L}-1\right)\right]\right\}\right) \rho(t)$

and following the one-photon derivation of Ref. [19] we take a time interval $\Delta t$ such that $r \Delta t \gg 1$

or, in other words, $\Delta t$ is chosen such that many atoms excited to the state $|c\rangle$ pass through the cavity during this time interval. This is necessary in order that the stepwise change of the cavity field can be treated as a continuous process. Then we may write [19]

$$
\frac{\Delta \rho}{\Delta t} \approx \frac{r}{p} \ln \left\{M_{G}\left[1+p\left(M_{L}-1\right)\right]\right\} \rho(t)
$$

if

$$
\frac{r}{p} \Delta t\left|\ln \left\{M_{G}\left[1+p\left(M_{L}-1\right)\right]\right\} \rho(t)\right| \ll 1 .
$$

In order to assume that Eq. (B3) is valid, the sufficient conditions are

$$
\left|\left[M_{L}\left(t_{\text {int }}\right)-1\right] \rho(t)\right| \ll 1
$$

and (for a general $\rho$ )

$$
\left|\ln \left[M_{G}\left(t_{\mathrm{at}}\right)\right] \rho(t)\right| \ll 1 .
$$

The condition (B6) requires that the change in the field density matrix due to the passage of one two-photon absorbing atom is small. In order to analyze condition (B7) we write

$$
\begin{aligned}
\ln \left\{1+\left[M_{G}(p / r)-1\right]\right\} \rho(t)= & {\left[M_{G}(p / r)-1\right] \rho(t) } \\
& +\left[M_{G}(p / r)-1\right]^{2} \rho(t)+\cdots .
\end{aligned}
$$

If we assume $K_{G} p / r \ll 1$, then

$$
\begin{aligned}
{\left[M_{G}(p / r)\right] \rho(t)=} & {\left[1-\frac{i}{\hbar} \frac{p}{r} V_{G}-\frac{1}{2 \hbar^{2}} \frac{p^{2}}{r^{2}} V_{G}\right] \rho(t) } \\
& \times\left[1+\frac{i}{\hbar} \frac{p}{r} V_{G}-\frac{1}{2 \hbar^{2}} \frac{p^{2}}{r^{2}} V_{G}\right]+\cdots
\end{aligned}
$$

Therefore,

$$
\begin{aligned}
\ln \left\{1+\left[M_{G}(p / r)-1\right]\right\} \rho(t)= & -\frac{i}{\hbar} \frac{p}{r}\left[V_{G}, \rho(t)\right] \\
& +\frac{1}{2 \hbar^{2}} \frac{p^{2}}{r^{2}}\left[\rho(t) V_{G}^{2}+V_{G}^{2} \rho(t)\right] \\
& -\frac{i}{\hbar} \frac{p^{2}}{r^{2}} V_{G} \rho V_{G} .
\end{aligned}
$$

If $p / r=R^{-1} \ll 1$ and $K_{G} p / r \ll 1$ we see that condition (B7) is fulfilled.

We note that for Poissonian pumping $(p \rightarrow 0)$ condition (B7) is necessarily held and we need to retain only the first term on the right-hand side of Eq. (B10), as we shall see below. In Sec. $\mathrm{V}$ we interpret the quantity $r \Delta t$ as the probability of making a measurement and finding an atom in the 
time interval $[t, t+\Delta t]$ and we shall take $r \Delta t \ll 1$. Of course, in this case Eq. (B5) can be satisfied even if Eqs. (B6) and (B7) are not.

Under the assumptions (B6) and (B7) and approximating the coarse-grained $\Delta \rho / \Delta t$ by the time derivative $d \rho / d t$, we obtain the master equation

$$
\frac{d \rho}{d t}=\frac{r}{p} \ln \left\{M_{G}\left[1+p\left(M_{L}-1\right)\right]\right\} \rho(t) .
$$

Now, following the procedure as in Ref. [19] we get

$$
\frac{d \rho}{d t}=\frac{r}{p} \ln \left[M_{G}\right] \rho(t)+\frac{r}{p} \ln \left[1+p\left(M_{L}-1\right)\right] .
$$

If we neglect terms proportional to $p^{2}$ and higher power terms in Eq. (B10), we obtain $[18,19]$

$$
\frac{d \rho}{d t}=-\frac{i}{\hbar}\left[V_{G}, \rho(t)\right]+\frac{r}{p} \ln \left[1+p\left(M_{L}-1\right)\right] \rho(t) .
$$

This is the master equation that describes the competition between two-photon parametric pumping and two-photon absorption by a stream of three-level atoms. We should stress that if we assume $r \Delta t \ll 1$ we can relax the assumption of small Rabi angles for the two-photon absorbing atoms.

Now let us review some of the approximations involved in the derivation of the above master equation. We have approximated the coarse-grained quotient in Eq. (B4) by the time derivative where $\Delta t$ is such that several atoms in the state $|c\rangle$ pass through the cavity during this interval of time, while the field reduced density matrix does not change ap- preciably. This approximation can lead to incorrect results regarding the time evolution (or dynamic behavior) of the system. However, for the steady state it is easy to show that such a differential equation yields the correct result [19]. We have assumed that $M_{G}$ and $M_{L}$ act independently over the time $p / r=t_{\text {at }}$ or, as the atoms spend a time $t_{\text {int }} \ll t_{\text {at }}$ in the cavity, we have assumed that $M_{G}$ and $M_{L}$ act independently over the time $t_{\text {int }}$. Such an approximation holds exactly for Poissonian statistics for the injection of absorbing atoms because

$$
\begin{aligned}
& {\left[\left(M_{G}-1\right), p\left(M_{L}-1\right)\right] \rho(t)} \\
& \quad=-\frac{i}{\hbar} \frac{p^{2}}{r}\left(\left[V_{G}, \widetilde{\rho}(t)\right]-\frac{1}{r}\left(M_{L}-1\right)\left[V_{P}, \rho(t)\right]\right)+\cdots,
\end{aligned}
$$

where $\tilde{\rho}(t)=\left(M_{L}-1\right) \rho(t)$ and we have used $\left(M_{G}\right.$ -1) $\rho(t) \approx-(i / \hbar)(1 / r)\left[V_{G}, \widetilde{\rho}(t)\right]$. We can do the same for other commutators that show up when we expand Eq. (B11) [19]. Therefore, as $r=p R$,

$$
\begin{aligned}
\frac{d \rho}{d t}= & -\frac{i}{\hbar}\left[V_{G}, \rho(t)\right]+r\left(M_{L}-1\right) \rho(t) \\
& +(\text { terms proportional to } p) .
\end{aligned}
$$

Taking the limit $p \rightarrow 0$, Eq. (B13) becomes the master equation for Poissonian pumping statistics discussed in the text as Eq. (36). It is valid for $K_{G} p / r \ll 1$ and holds irrespective of the magnitude of the Rabi angle of the two-photon absorption since, taking $K_{G}=0\left(M_{G}=1\right), p \rightarrow 0$ guarantees that Eq. (B5) is fulfilled.
[1] H. P. Yuen, Phys. Rev. A 13, 2226 (1976).

[2] See, e.g., the review by R. Loudon and P. L. Knight, J. Mod. Opt. 34, 709 (1987).

[3] H. D. Simaan and R. Loudon, J. Phys. A 8, 539 (1975); 11, 435 (1978); N. Tornau and A. Bach, Opt. Commun. 11, 46 (1974); G. S. Agarwal and G. P. Hildred, ibid. 58, 287 (1986); S. Charturverdi, P. Shanta, and V. Srinivasan, ibid. 78, 289 (1990); L. Gilles and P. L. Knight, Phys. Rev. A 48, 1582 (1993).

[4] G. S. Agarwal, Phys. Rev. A 1, 1445 (1970); R. Loudon, The Quantum Theory of Light (Clarendon, Oxford, 1973); K. J. McNeil and D. F. Walls, J. Phys. A 7, 617 (1974).

[5] E. E. Hach III and C. C. Gerry, Phys. Rev. A 49, 490 (1994); C. C. Gerry and E. E. Hach III, Phys. Lett. A 174, 185 (1993); C. C. Gerry, J. Mod. Opt. 40, 1053 (1993); V. Bužek and P. L. Knight, in Progress in Optics, edited by E. Wolf (NorthHolland, Amsterdam, 1995), Vol. XXXIV, p. 1.

[6] L. Gilles, B. M. Garraway, and P. L. Knight, Phys. Rev. A 49, 2785 (1994).

[7] G. Rempe, R. J. Thompson, H. J. Kimble, and R. Lalezari, Opt. Lett. 17, 363 (1992)

[8] See the review by B. W. Shore and P. L. Knight, J. Mod. Opt. 40, 1195 (1993), and references therein.
[9] D. Meschede, H. Walther, and G. Muller, Phys. Rev. Lett. 54, 551 (1985); G. Rempe and H. Walther, ibid. 58, 353 (1987); M. Brune, J. M. Raimond, P. Goy, L. Davidovich, and S. Haroche, ibid. 59, 1899 (1987).

[10] Kyungwon An, J. J. Childs, R. R. Dasari, and M. S. Feld, Phys. Rev. Lett. 19, 3375 (1994).

[11] See, for example, R. Blatt, J. I. Cirac, and P. Zoller, Phys. Rev. A 52, 518 (1995); J. F. Poyatos, J. I. Cirac, R. Blatt, and P. Zoller, ibid. 54, 1532 (1996); A. W. Vogt, J. I. Cirac, and P. Zoller, ibid. 53, 950 (1996); S.-C. Gou and P. L. Knight, ibid. 54, 1682 (1996); S.-C. Gou, J. Steinbach, and P. L. Knight, ibid. 54, R1014 (1996); R. L. de Matos Filho and W. Vogel, Phys. Rev. Lett. 76, 608 (1996); W. Vogel and R. L. de Matos Filho, Phys. Rev. A 52, 4214 (1995).

[12] J. F. Poyatos, J. I. Cirac, and P. Zoller, Phys. Rev. Lett. 77, 4728 (1996).

[13] C. Monroe, D. M. Meekhof, B. E. King, and D. J. Wineland, Science 272, 1131 (1996); D. M. Meekhof, C. Monroe, B. E. King, W. M. Itano, and D. J. Wineland, Phys. Rev. Lett. 76, 1796 (1996).

[14] H. J. Carmichael, in An Open Systems Approach to Quantum Optics, Lecture Notes in Physics Vol. m18 (Springer-Verlag, Berlin, 1993). 
[15] J. Dalibard, Y. Castin, and K. Mфlmer, Phys. Rev. Lett. 68, 580 (1992); K. Mølmer, Y. Castin, and J. Dalibard, J. Opt. Soc. Am. B 10, 524 (1993); G. C. Hegerfeldt, and T. S. Wilser, in II International Wigner Symposium, 1991, edited by H. D. Doebner et al. (World Scientific, Singapore, 1992); see also P. L. Knight and B. M. Garraway, Quantum Dynamics of Simple Systems, Proceedings of the 44th Scottish Universities Summer School in Physics, edited by G.-L. Oppo et al. (Institute of Physics, Bristol, 1996), p. 199; M. B. Plenio and P. L. Knight, Rev. Mod. Phys. (to be published).

[16] M. Sargent III, M. O. Scully, and W. Lamb, Jr., Laser Physics (Addison-Wesley, Reading, MA, 1974).

[17] See, for example, P. Filipowicz, J. Javanainen, and P. Meystre, Phys. Rev. A 34, 3077 (1986); L. Davidovich, J. M. Raimond,
M. Brune, and S. Haroche, ibid. 36, 3771 (1987); U. Herzog, ibid. 50, 783 (1994).

[18] J. Bergou, L. Davidovich, M. Orszag, C. Benkert, M. Hillery, and M. O. Scully, Opt. Commun. 72, 82 (1989); Phys. Rev. A 40, 5073 (1989); J. Bergou and P. Kálmán, ibid. 43, 3690 (1991); E. S. Guerra, A. Z. Khoury, L. Davidovich, and N. Zagury, ibid. 44, 7785 (1991); J. D. Cresser, ibid. 46, 5913 (1992).

[19] L. Davidovich, S.-Y. Zhu, A. Z. Khoury, and C. Su, Phys. Rev. A 46, 1630 (1992).

[20] G. Lindblad, Commun. Math. Phys. 48, 119 (1976).

[21] B. M. Garraway and P. L. Knight, Phys. Rev. A 49, 1266 (1994). 